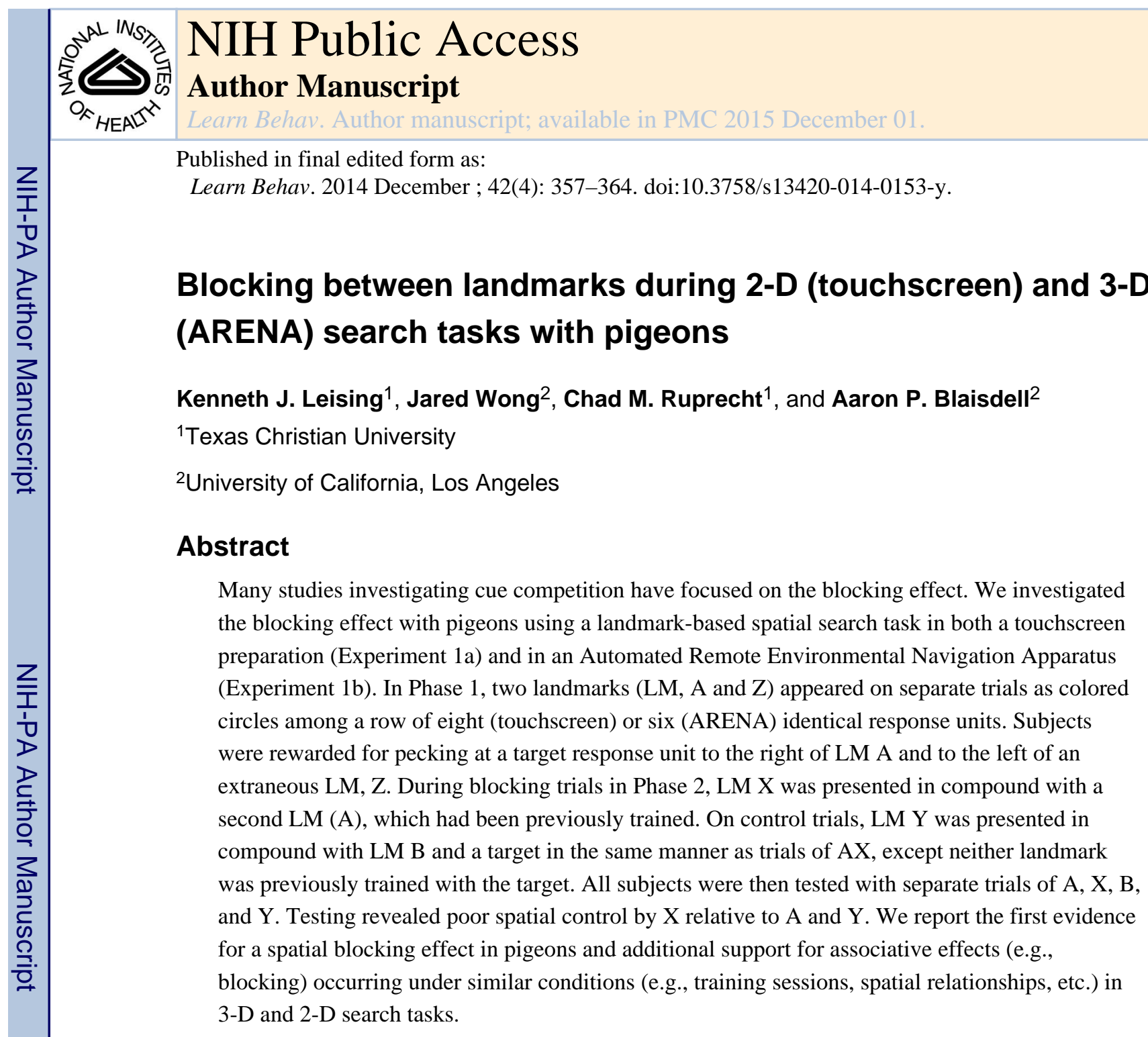

\title{
Keywords
}

blocking; spatial learning; cue competition; navigation; generalization

\begin{abstract}
Most vertebrate species navigate their environment for a variety of reasons, such as to search for food, find mates, and avoid predators. Such movement often necessitates that the animal keep track of where it has been, where it is going, and how to return home. One method of doing this is to learn to use spatial cues, such as beacons and landmarks (Leising \& Blaisdell, 2009). Spatial learning has been demonstrated in virtually all vertebrates studied, but the learning process itself has been less well characterized. While some argue for specialized spatial learning systems or modules (Cheng, 1988; O'Keefe \& Nadel, 1978), others have suggested that a more general-purpose associative learning processes may govern spatial learning (e.g., Leising \& Blaisdell, 2009; Miller \& Shettleworth, 2007). This
\end{abstract}

Correspondence concerning this article should be addressed to Kenneth J. Leising, Department of Psychology, Texas Christian University, 2800 S. University Dr., Box 298920, Fort Worth, TX 76129. k.j.leising @ tcu.edu.

Kenneth J. Leising, Department of Psychology, Texas Christian University; Jared Wong, Department of Psychology, University of California, Los Angeles; Chad M. Ruprecht, Department of Psychology, Texas Christian University; Aaron P. Blaisdell, Department of Psychology, University of California, Los Angeles. 
latter proposal has been supported through the search for commonalities between spatial learning and more conventional associative learning paradigms, such as Pavlovian and instrumental conditioning.

The blocking effect is a hallmark of an associative learning process (Kamin, 1969; Moore \& Schmajuk, 2008). In blocking, cue A, such as a conditioned stimulus or CS, is trained as a predictor of an outcome, such as an unconditioned stimulus or US. After CS A has acquired a conditioned response through pairings with the US, additional A-US pairings are given in the presence of another CS (X). That is, the subject first receives A-US pairings, followed by AX-US pairings. When the subject is subsequently tested on $\mathrm{X}$ alone, much less conditioned responding is observed relative to a control group. One type of control group used in a between-subjects demonstration of blocking is to omit Phase 1 A-US pairings and only give AX-US pairings. Responding to $\mathrm{X}$ at test is greater in the Control than in the Blocking group for which A had been pre-trained prior to AX-US pairings. A second control consists of a within-subject design, with the subject receiving A-US pairings in Phase 1 of training, and then AX-US pairings alternated with BY-US pairings in Phase 2 of training. Then subjects are tested separately on $\mathrm{X}$ and $\mathrm{Y}$. Greater conditioned responding to $\mathrm{Y}$ than to $\mathrm{X}$ indicates that conditioning to $\mathrm{X}$ was blocked by pre-training of $\mathrm{A}$; whereas $\mathrm{Y}$ was not blocked by B which had no pre-training. In a spatial task, a landmark acts as a CS that reliably predicts an upcoming US, but additionally, provides stable information regarding the location of the US (e.g., Ruprecht, Quintana, Wolf, \& Leising, in press). Conventional blocking is evaluated in terms of the magnitude of responding elicited by the blocked cue; however, spatial blocking is evaluated in terms of the spatial accuracy. Blocking of spatial control by a landmark has been demonstrated in rats using arrays of landmarks in the openfield (Biegler \& Morris, 1999; Stahlman \& Blaisdell, 2009), as well as using extra-maze cues in a Morris pool (Rodrigo et al., 1997) and radial-arm maze (Chamizo et al., 1985). No studies demonstrating spatial blocking have been reported with an avian species.

There is reason to believe pigeons are likely to show evidence of spatial blocking. Pigeons' spatial abilities have been extensively studied, and laboratory tasks show that pigeons readily learn to use a landmark to find a hidden target (e.g., a food goal; e.g., Cheng \& Spetch, 1995). Evidence has also been found with pigeons for spatial overshadowing, in which learning of more distal spatial landmarks was reduced by the presence of more proximal landmarks (Leising, Garlick, \& Blaisdell, 2011; Spetch, 1995). We recently reported two spatial overshadowing experiments using operant training in either a touchscreen chamber (Experiments 1 and 2) or an automated open-field ARENA (Experiment 2, Leising et al., 2011). These results suggest that associative processes govern spatial learning, and that these processes operate similarly in 3-D (ARENA) and 2-D (touchscreen) tasks. The current studies investigating the blocking effect borrowed heavily from those previously used in our laboratory to demonstrate spatial overshadowing (Leising et al., 2011).

Experiments 1a and $1 \mathrm{~b}$ use a within-subject design, in which subjects learn in Phase 1 of training to locate hidden targets by using landmarks as discriminative spatial cues. Previous studies investigating blocking in appetitive conditioning have found a considerable degree of generalization between stimuli (e.g., Ganesan and Pearce, 1988). In the current experiments, 
we trained pigeons to respond at a target to the left of one landmark and to the right of another in Phase 1. This type of discrimination training should attenuate the presence of any side bias, as well as attenuate generalization across landmarks by requiring subjects to learn about the qualities of each landmark. In Phase 2, all birds searched for the target in the presence of a compound of LM A and a novel LM X on some trials (AX), and other trials in the presence of a compound of two novel LMs (BY). Following Phase 2 acquisition, pigeons receive nonreinforced probe tests of each landmark separately. If pretraining of A blocks spatial control by $\mathrm{X}$, then we expect to see poorer spatial control by $\mathrm{X}$ at test compared to $\mathrm{A}$ and, more critically, compared to Y.

\section{Experiments $1 \mathrm{a}$ and $1 \mathrm{~b}$}

Experiments $1 \mathrm{a}$ and $1 \mathrm{~b}$ also investigated whether a blocking effect observed with a 2-D touchscreen task (Experiment 1a) would also be observed using identical experimental parameters within a "real-space" three-dimensional (3-D) task (Experiment 1b). Though differences have been found in the pattern of spatial control by landmarks in a touchscreenequipped chamber and open field (e.g., Lechelt \& Spetch, 1997), most studies have revealed more similarities than differences (e.g., Spetch, Cheng, \& MacDonald, 1996; Leising et al., 2011). We recently reported analogous findings on the overshadowing effect with pigeons in a touchscreen-equipped chamber and an automated remote environmental navigation apparatus (ARENA, i.e., an automated open field; Leising, Garlick, \& Blaisdell, 2011, see Leising, Garlick, Paranteau, \& Blaisdell, 2009 for more details on ARENA). ARENA allows subjects to travel through open space to interact with landmarks and response units, and thus provides a more ecologically valid 3-D search task in which to compare performance to the more commonly used touchscreen-equipped apparatus.

Pigeons in each apparatus received the same blocking procedure, but the method of response detection, stimulus delivery, and reward retrieval differed between apparatus. In Experiment 1a, pigeons pecked at a row of eight circular visual response units on a touchscreenequipped display (see Figure 1). The response unit locations were defined by a white border against a black background. Landmarks within the units were displayed as colored response units. In Experiment 1b, pigeons pecked at a row of six plastic ARENA response modules on the floor of an open field testing area (see Figure 1). The response unit locations were defined by a recessed well in the center of each module. Landmark modules were defined within the row of response units by colored lights generated by LEDs located within the recessed well of each module.

\section{Method}

Subjects-Five white Carneau pigeons (Columba livia; Double T Farm, IA) served as subjects in the touchscreen apparatus. They were naïve to the current procedure, array, and colored landmarks, but had previously participated in a previous touchscreen spatial-search tasks. Four white Carneau pigeons (Columba livia; Double T Farm, IA) served as subjects in ARENA. Subjects had previously served in conditional discrimination (Leising et al., 2009) and overshadowing (Leising et al., 2011) procedures in ARENA. Pigeons in Experiments 1a and $1 \mathrm{~b}$ were maintained at $85 \%$ of their free-feeding weights. They were individually 
housed in a colony with a 12-hr light-dark cycle, and were provided with free access to water and grit. Experimental procedures occurred during the light portion of the cycle.

Apparatus-Experiment 1a was conducted in a flat-black Plexiglas chamber $(38.0 \mathrm{~cm}$ wide $\times 36.0 \mathrm{~cm}$ deep $\times 38.0 \mathrm{~cm}$ high). All stimuli were presented by computer on the LCD monitor (NEC MultiSync LCD1550M) visible through a $23.2 \times 30.5 \mathrm{~cm}$ viewing window in the middle of the front panel of the chamber. The bottom edge of the viewing window was $13.0 \mathrm{~cm}$ above the chamber floor. Pecks to the monitor were detected by an infrared touchscreen (Carroll Touch, Elotouch Systems, Fremont, CA) mounted on the front panel. A 28-V houselight located in the ceiling of the box was illuminated at all times. A food hopper (Coulbourn Instruments, Allentown, PA) was located in the center of the front panel, its access hole flush with the floor. All experimental events were controlled and recorded with a Pentium III-class computer (Dell, Austin, TX). A video card controlled the monitor in the SVGA graphics mode $(800 \times 600$ pixels $)$.

A row of eight 2.0-cm in diameter units served as stimuli (see Figure 1a). All eight units were defined by a white border against a black background. The units were separated by 3.0 $\mathrm{cm}$ center to center, and the edges of the row were centered with respect to the monitor. A response area was defined by an invisible border that extended $2.0 \mathrm{~mm}$ beyond the visible border of each unit. A $2.0-\mathrm{mm}^{2}$ black square was centered within each unit. This black square was not present when a disk served as a landmark. A unit serving as a response unit was filled white to $35 \%$ of total possible brightness, or intensity. A unit serving as a landmark was filled with a texture and color. The colors were pure green, blue, and red, as well as orange (RGB: 255, 102, 51). The graphic textures were leaves (green), scales (blue), 3-D cubes (red), and interconnected rectangles (orange). Assignment of color to landmark was counterbalanced across birds.

Experiment $1 \mathrm{~b}$ was conducted in a sound-attenuating room with a $2.3 \times 1.5 \mathrm{~m}$ test area. ARENA included the testing room, six ARENA modules, and a holding cage with grain hopper. A white noise generator provided background noise $(56-63 \mathrm{~dB}(\mathrm{~A}))$ in the testing area. A Sony SSC-DC374 color video camera with wide-angle lens was mounted on the ceiling $2.4 \mathrm{~m}$ above the floor of the test area and allowed for viewing and recording of experimental procedures in an adjacent room. A $31.8 \times 16.7 \mathrm{~cm}$ automated pet door was mounted one wall of the testing room and led to a holding cage with a hopper identical to that described in the touchscreen task mounted on the rear wall. The walls, floor, and ceiling of the holding cage were made of white acrylic, and the holding cage was illuminated by a 28-V Med Associates houselight.

Stimulus presentation and response detection was accomplished by the modules (Badelt \& Blaisdell, 2008; Leising et al., 2009; see Figure 1a). Each module was a square plastic box measuring $12.9 \mathrm{~cm}$ wide and $3.5 \mathrm{~cm}$ high. A well measuring $3.0 \mathrm{~cm}$ diameter by $3.0 \mathrm{~cm}$ was created in the center of the module. A sensor circuit, including sensing and reference electrodes, was enclosed within the module. The sensing field was restricted to the volume within the cup (described in more detail in Badelt \& Blaisdell, 2008). The sensor was activated when a pigeon's beak (or any conductive mass) broke the top planar surface and entered the space within the well. The module also housed a transmitter and receiver, which 
communicated with a PC computer in an adjacent room using radio waves. The network allowed each module to receive command signals, change the status of the LEDs, and send detection events to the computer. Each module was equipped with six LEDs located in the bottom of the response area. Three of the bulbs were positioned horizontally, and the remaining bulbs were positioned vertically facing up towards the opening of the well. The vertical bulbs were used to present colored lights to serve as visual landmarks. The response and stimulus areas of a module were defined by the perimeter of the recessed well. Six modules served as response units positioned in a single row, and spaced $31.4 \mathrm{~cm}$ center to center. The midpoint of the six units was centered at an imaginary line perpendicular to the food hopper in the holding cage. All units were $100.3 \mathrm{~cm}$ from the south wall of the test area and $49.5 \mathrm{~cm}$ from the north wall.

The colors used within the modules were sky blue (RGB: 102, 255, 255), lime green (RGB: 153, 255, 000), magenta (RGB: 153, 51, 255), orange (RGB: 255, 102, 51), and firebrick (RBG: 178, 34, 34). The color for $\mathrm{Z}$ was orange for each subject. Assignment of color to landmark for the remaining colors was counterbalanced across birds.

Procedure-All procedural details (Phase 1, Phase 2, and Test) were the same for the touchscreen and ARENA preparations, with the exception of the use of 3-D modules as response locations and food retrieval from a separate area in ARENA. All subjects had been previously trained to retrieve mixed grain and pellets from a food hopper. At the beginning of the experiment, pigeons were trained to search for a target unit among the other response units. On each trial, one unit was randomly selected without replacement to be the target and filled white at full brightness (RGB: 255, 255, 255). Pecks to all response units were recorded during a trial. Search training progressed in stages during which we manipulated both the schedule of reinforcement and the brightness of the target (See Leising et al., 2011 for details). The schedule of reinforcement increased across stages from continuous (CRF), to a modified fixed-ratio 2 (FR-2), which required two consecutive pecks to the target; and finally, to an FR-2 followed by a fixed-interval (FI) of $8 \mathrm{~s}$. The FI period was initiated after the second consecutive peck to the target (thereby meeting the FR-2 criterion) and the trial terminated with reinforcement after one additional peck to the target following the 8-s interval. The brightness of the target was progressively dimmed across training from full brightness to $78 \%$, and then $61 \%$. During this portion of training, the target unit was always brighter than the non-target response units, which were lit to $35 \%$ brightness. Subjects advanced to a new reinforcement schedule and a dimmer target disk with ten consecutive correct trials, or regressed with five consecutive incorrect trials. Sessions lasted for 72 trials or 60 minutes. All trials were followed by a variable-time (VT) $15 \mathrm{~s}$ intertrial interval (ITI). One session with $80 \%$ of trials ending in reinforcement was required to advance to landmark training.

Phase 1: All subjects were trained with two landmarks with stable spatial relationships to a hidden target in Phase 1. On A+trials, the target was located one unit to the right of A, whereas, the target was located one unit to the left of $Z$. Each session consisted of 80 trials (40 trials of $A$ and $Z$ ). The position of the target was selected pseudorandomly from units 27 in Experiment 1a and 2-5 in Experiment 1b, thereby determining the location of $\mathrm{A}$ or $\mathrm{Z}$. 
There was an unstable relationship between the location of the target and other cues in the operant box (e.g., frame of the display), but the spatial relationship between the landmark and the target remained stable across trials. Initially, the target was filled white at full brightness and pecks were reinforced according to a FR-2 + variable interval (VI) 4-s schedule of reinforcement. In the variable-interval schedule, the duration of the interval was randomly selected from a range of $1-7 \mathrm{~s}$ (in steps of $1 \mathrm{~s}$ ). Trials terminated with the requisite number of pecks at the target, 30 cumulative incorrect pecks to any unit, or after $60 \mathrm{~s}$. All trials were followed by a VT-15 s ITI. Each session consisted of 80 trials (40 trials of A and $\mathrm{Z}$ intermixed) or terminated after 60 minutes, whichever came first.

As in search training, the target was progressively dimmed within and across sessions to $78 \%, 61 \%$, and finally $35 \%$ brightness; at which point it was indistinguishable from the rest of the response units. With the target and non-target locations at $35 \%$ brightness, the schedule of reinforcement was concurrently increased from CRF, to a modified FR-2, and finally to an FR-2 + VI-4 s. Subjects advanced to a new reinforcement schedule and a dimmer target disk with ten consecutive correct trials, or regressed with five consecutive incorrect trials. Each subject was advanced independently to Phase 2 after it completed two consecutive sessions in which the FR-2 + VI-4 reinforcement criterion was met on $80 \%$ of trials and the mean proportion of pecks at the target (pecks at the target location divided by all responses) was greater than or equal to .60 .

Phase 2: All subjects received 40 compound trials of A paired with a novel LM, X (AX+) intermixed with 40 compound trials of two novel LMs, B and Y (BY+) in each session. The position of A relative to the target was identical to Phase 1. During AX trials, $X$ was positioned at the location on the opposite side of the target from A; the same distance from the target as A, but in the opposite direction. LMs B and $\mathrm{Y}$ were positioned in the same manner as $\mathrm{A}$ and $\mathrm{X}$, respectively. As in previous training, the target was progressively dimmed across training to $78 \%, 61 \%$, and finally $35 \%$ brightness. The schedule of reinforcement was also increased from CRF, to a modified FR-2, and finally to an FR-2 + VI 4-s. Trials terminated with the requisite number of pecks at the target, 30 cumulative incorrect pecks to any unit, or after $60 \mathrm{~s}$. All trials were followed by a VT $15 \mathrm{~s}$ ITI. Sessions were 80 trials or 60 minutes, whichever came first. Each subject was advanced independently to Test after the reinforcement criterion was met on $80 \%$ of trials and the mean proportion of pecks at the target (pecks at the target location divided by all responses) was greater than or equal to .60 .

Test: All subjects received two test sessions with three non-reinforced test trials of LMs X, $\mathrm{A}, \mathrm{B}$ and $\mathrm{Y}$ alone in each test session. Each test session consisted of 44 trials, with 16 reinforced trials of AX and BY as in Phase 2 (32 total baseline trials) intermixed with 12 test trials. On a test trial, the second peck of the trial initiated an 8-s interval, after which the trial terminated without reinforcement (c.f., Leising et al., 2011; Spetch, 1995). Test trials automatically terminated after $30 \mathrm{~s}$ if subjects failed to meet the response requirement. The order of trials and the position of the target on each trial were counterbalanced across subjects. During testing, the target location was always selected to be either position 4 or 5 in Experiment 1a and position 3 or 4 in Experiment $1 \mathrm{~b}$. 
Measures: The difference between the proportion of pecks at the target-side (predicted target) and opposite-side (generalization target) of the landmark during each trial was calculated as a measure of spatial control by each landmark. We did this to differentiate between spatial control of each cue as a landmark on the one hand, and as a beacon on the other (cf. Mackintosh, 2002). The proportion of pecks at the predicted and generalization target during each test trial was calculated by dividing the number of pecks at the predicted or generalization target by the number of pecks at all of the locations. To calculate a difference score, we then subtracted the proportion of pecks at the generalization target from the proportion of pecks at the predicted target. The difference from 0 indicates the strength of spatial control (i.e., larger values indicating greater spatial control), and the sign of the value indicates the direction of control (i.e., positive values indicate more responses in the direction of the predicted goal).

\section{Results and Discussion}

In the touchscreen preparation, one subject failed to complete Phase 1 after 20 additional sessions of training ( $n=4$ in Experiment 1a). In ARENA, during testing one of the modules (module 4) became unresponsive (e.g., no illumination or response detection) for the entire duration of a test session for one bird ( $n=3$ in Experiment 1b). All analyses are reported with the remaining subjects combined and Apparatus included as a between-subjects factor $(N=7)$. The mean number of training sessions required to advance to testing in Phase 1 (ARENA $M=14.7, S D=3.1$; Touchscreen $M=17.5, S D=7.23$ ) and Phase 2 (ARENA $M$ $=3.7, S D=2.08$; Touchscreen $M=4.5, S D=2.4$ ) were similar in both procedures (see Figure 2). In Experiment 1a, the reinforcement criterion was met on $94 \%(S D=2.80)$ of trials during Phases 1 and 2 and the mean proportion of pecks at the target increased from . $32(S D=.04)$ during the first session of Phase 1 to $.70(S D=.16)$ during the last session. The reinforcement criterion was met on $91 \%(S D=1.18)$ of trials during test sessions. In Experiment $1 \mathrm{~b}$, the criterion was met on $98 \%(S D=.30)$ of trials during training and testing, and the mean proportion of pecks at the target increased from .22 $(S D=.11)$ during the first session of Phase 1 to $.60(\mathrm{SD}=.03)$ during the last session.

Figure 3 displays the mean difference score for each landmark at test. LM A exerted greater spatial control than $\mathrm{X}$, with $\mathrm{B}$ and $\mathrm{Y}$ demonstrating levels of spatial control intermediate to that of A and X. A $2 \times 2 \times 2$ mixed model ANOVA conducted on difference scores with Apparatus (Touchscreen or ARENA) as a between-subjects factor and Condition (Blocking and Control) and Landmark ( $\mathrm{A} / \mathrm{B}$ or $\mathrm{X} / \mathrm{Y})$ as repeated measure factors revealed a main effect of Condition, $F(1,5)=7.55, p=.04, \eta^{2}=.60$, Landmark, $F(1,5)=22.51, p=.005, \eta^{2}=$. 82 , and the interaction of Condition x Landmark, $F(1,5)=12.60, p=.02, \eta^{2}=.72$, but did not find a main effect of Apparatus or the interaction of Apparatus with any other factor. Although the comparison is underpowered (i.e., small samples), the data in Figure 3 indicate very similar patterns of responding across experiments and a power analysis using the RMSEE of the three-way interaction indicated 225 subjects would be required to detect the interaction. We then collapsed across Apparatus and conducted planned comparisons to isolate the source of the interaction. Spatial control by A was greater than by $\mathrm{X}, F(1,6)=$ $32.21, p=.001, \eta^{2}=.84$, and $\mathrm{B}, F(1,6)=8.14, p=.03, \eta^{2}=.58$. There was no difference between spatial control by B versus $\mathrm{Y}, F<1.0$. Critically, spatial control by $\mathrm{X}$ was poorer 
than by $\mathrm{Y}$, indicating the blocking effect, $F(1,6)=12.31, p=.01, \eta^{2}=.67$. We compared the difference scores for each landmark to zero using single-samples $t$-tests, which revealed that all landmarks exerted reliable spatial control over responding, $t \mathrm{~s}(5)>4.28, p \mathrm{~s}<.01, d \mathrm{~s}$ $>1.51$.

A pilot experiment revealed that training only on LM A in Phase 1 resulted in a large amount of generalization to the other landmarks during test. Training with two landmarks in Phase 1 of the current experiments, such that correct responses were required to the left and right of a landmark reduced potential generalization from A and increased spatial control by each landmark. Evaluation of spatial control revealed a clear blocking effect. Training of spatial control by LM A in Phase 1 resulted in blocking of spatial control by X. LM Y, however, did not show diminished spatial control compared to $\mathrm{B}$, which did not receive prior training. This is, to our knowledge, the first clear demonstration of spatial blocking in a touchscreen or open-field preparation with pigeons. This extends the evidence for cue competition phenomena, such as overshadowing, in spatial learning, providing further evidence for the operation of associative processes in spatial learning. Interestingly, we also observed stronger spatial control by LM A than B. This difference was likely due to more training of $A$ (Phase 1 and 2) compared to training with B (Phase 2 only); however, we would also expect reciprocal-overshadowing between $\mathrm{B}$ and $\mathrm{Y}$ during Phase 2 training to lessen spatial control by B alone compared to A, which received trials of A alone during Phase 1. Additional control groups would be needed to determine which factor was responsible for the difference between $\mathrm{A}$ and $\mathrm{B}$.

One concern with Experiment $1 \mathrm{~b}$ may be that subjects did not detect the presence of $\mathrm{X}$ during Phase 2 in the ARENA preparation. With a distance of $34 \mathrm{~cm}$ between the target and LM X in ARENA, perhaps the pigeons failed to detect that $X$ was present thereby producing a blocking-like effect due to a complete failure of acquisition. Figure 4a displays the mean distribution of searches across the target location and the two response units to the left and right of the target on trials of A during the last trial of the last session of Phase 1 and from the first trial of AX during the first session of Phase 2. The number of responses at each unit was standardized across trials, such that responses were recorded relative to the expected target on each trial. From the last trial of A during Phase 1 to the first trial of AX, we would expect the most change at the response unit to the right of $\mathrm{X}$ where the subjects may search as a result of generalization from A. Figures $4 \mathrm{a}$ and $4 \mathrm{~b}$ reveal several changes, including more responses at the generalization target location (1) during AX trials in Figure 4a. By the last trial of $\mathrm{AX}$, responding at the generalization target had decreased. These changes suggest that 1) subjects detected the new landmark, and 2) subjects explored the environment enough to detect any new landmarks or targets. Consequently, the opportunity to learn about the value of $\mathrm{X}$ was present during training.

\section{General Discussion}

Pigeons were trained to peck at a target location signaled by two landmarks ( $\mathrm{A}+$ and $\mathrm{Z}+$ ) in a touchscreen-equipped chamber (Experiment 1a) or within an automated 3-D open field (ARENA, Experiment 1b). In the second phase, an additional landmark (X) was presented in compound with trials of $\mathrm{A}(\mathrm{AX}+)$ and the target. On separate trials, pecks to a target were 
reinforced in the presence of a second pair of novel landmarks (BY+) positioned in the same relationship to the target as $\mathrm{AX}$. Test trials of each landmark alone were presented. Critically, spatial control by X was poor relative to A and Y. The results of Experiment 1a and $1 \mathrm{~b}$ revealed no differences in the magnitude or pattern of spatial control between the touchscreen and ARENA search tasks. These results provide the first evidence for the blocking effect with pigeons in the spatial domain.

After training with BY and the target during Phase 2 of Experiments 1a and 1b, test trials of $\mathrm{B}$ and $\mathrm{Y}$ alone revealed a larger proportion of responses at the predicted target from training than the generalization target. This was crucial, as more responding at the target location in the presence of each landmark required learning to respond in different directions from LMs $\mathrm{B}$ and $\mathrm{Y}$. This result indicates that subjects learned the B-target and Y-target spatial relationships and were not simply generalizing from other landmarks encountered during training (e.g., LMs A and Z). There was also evidence for learning of the X-target spatial relationship in Experiment 1a and 1b, but the key finding was that less spatial control was observed in the presence of $\mathrm{X}$ than $\mathrm{Y}$, which was not trained in the presence of an already established landmark.

The results from these experiments converge with a growing body of evidence suggesting cue competition effects in the spatial domain are a subset of a more general associative learning mechanism. Rodrigo et al. (1997) trained rats in a Morris pool to locate and swim to a hidden platform. Landmarks hanging over the perimeter of the pool were provided as extra-maze cues. Rodrigo et al. first verified that rats placed on the hidden platform in full view of four landmarks for several trials were subsequently able to locate the hidden platform in the presence of only three landmarks. Rats in the blocking group were placed on the platform with landmarks A, B, and C suspended above the edge of the pool, whereas rats in the control group received identical trials but with a different set of landmarks (i.e., $\mathrm{A}^{\prime}$, B ', and $\mathrm{C}^{\prime}$ ). All groups then received four days of placement trials with the configuration of LMs A, B, C, and X. At test, rats were placed in the pool to find the hidden target with only a subset of landmarks (e.g., A, B, and X) and performance in the blocking group showed a significant deficit relative to the control group. All groups were equally capable of predicting the quadrant of the hidden platform based on LMs A, B, and C or A, B, C, and X, but the blocking group was deficient in preferring the quadrant of the platform signaled by the subset of LMs A, B, and X (but see Rodrigo, Chamizo, \& Mackintosh, 2013, for the effects of sex differences). Similarly, humans trained to navigate a computerized version of a virtual pool with one set of landmarks performed poorly when tested on a second set of added landmarks (Hamilton \& Sutherland, 1999), and honeybees trained to find reward in the presence of one cue fail to show spatial control by a subsequently added cue (Cheng \& Spetch, 2001).

More recently, Stahlman and Blaisdell (2009) were able to capture spatial blocking among landmarks in an open-field foraging task in rats. The open-field featured a $4 \times 4$ array of response cups, with food being buried under a single target cup. Adjacent landmarks could be placed to the left or right of the target cup. In Phase 1, rats were presented with A+ trials during which LM A signaled the location of hidden food. In Phase 2, rats were given AX+ trials in which $\mathrm{X}$ served as a redundant spatial cue to the location of food. Additionally, BY 
+ trials were given as a within-subjects overshadowing-control procedure. At test, rats received non-reinforced presentations of $\mathrm{X}$ and $\mathrm{Y}$ on separate trials. Rats took longer to find the training target location in the presence of $\mathrm{X}$ than $\mathrm{Y}$, thereby demonstrating that spatial control by $\mathrm{X}$ was blocked by prior learning with $\mathrm{A}$. Obtaining spatial blocking in an avian model has not been reported with an open field or touchscreen apparatus.

The blocking effect is a hallmark of an associative process. Early attempts to characterize spatial learning suggested it was specialized and modular, sharing little with other types of learning (Cheng, 1988; O'Keefe \& Nadel, 1978). More recent characterizations identified two systems of spatial learning; landmark learning that is immune to associative effects (i.e., locale system) and beacon homing that is not (e.g., taxon system; O'Keefe and Nadel, 1978). Much evidence has been required to encourage thinking about landmark learning as another instance of associative learning. The current studies found a deficit in spatial accuracy in the presence of a blocked landmark closely resembling the deficit in the magnitude of responding reported in more conventional classical and operant conditioning tasks. These results add pigeons to the list of species demonstrating spatial blocking, which also includes humans (e.g., Hamilton \& Sutherland, 1999), rats (e.g., Biegler \& Morris, 1999; Chamizo et al., 1985; Rodrigo et al., 1997; Stahlman \& Blaisdell, 2009), and honeybees (e.g., Cheng \& Spetch, 2001).

\section{Acknowledgments}

Support for this research was provided by NIH Grant MH066855 (A.P. Blaisdell). This research was conducted following the relevant ethics guidelines for research with animals and was approved by UCLA and TCU's institutional IACUCs. A grant provided by the TCU Research and Creative Activities Fund supported the completion of this manuscript.

\section{References}

Badelt SW, Blaisdell AP. Capacitive sensors for detecting proximity and response. Behavior Research Methods. 2008; 40:613-621. [PubMed: 18522073]

Biegler R, Morris RG. Landmark stability is a prerequisite for spatial but not discrimination learning. Nature. 1993; 361:631-633. [PubMed: 8437622]

Chamizo VD, Sterio D, Mackintosh NJ. Blocking and Overshadowing between Intra-maze and Extrtamaze Cues: A test of the independence of locale and guidance learning. The Quarterly Journal of Experimental Psychology. 1985; 37B:235-253.

Cheng K. The determination of direction in landmark-based spatial search in pigeons: A further test of the vector sum model. Animal Learning \& Behavior. 1994; 22:291-301.

Cheng K, Spetch ML. Blocking in landmark-based search in honeybees. Animal Learning \& Behavior. 2001; 29:1-9.

Ganesan R, Pearce JM. Effects of changing the unconditioned stimulus on appetitive blocking. Journal of Experimental Psychology: Animal Behavior Processes. 1988; 14:280-291. [PubMed: 3404082]

Hamilton DA, Sutherland RJ. Blocking in human place learning: Evidence from virtual navigation. Psychobiology. 1999; 27:453-461.

Kamil AC, Cheng K. Way-finding and landmarks: The multiple-bearings hypothesis. Journal of Experimental Biology. 2001; 204:103-113. [PubMed: 11104714]

Kamin, LJ. Predictability, surprise, attention and conditioning. In: Campbell, BA.; Church, RM., editors. Punishment and Aversive Behavior. Appleton-Century-Crofts; New York: 1969. p. 276-296.

Lechelt DP, Spetch ML. Pigeons' Use of Landmarks for Spatial Search in a Laboratory Arena and in Digitized Images of the Arena. Learning and Motivation. 1997; 28:424-445. 
Leising KJ, Blaisdell AP. Associative basis of landmark learning and integration in vertebrates. Comparative Cognition \& Behavior Reviews. 2009; 4:80-102. [PubMed: 20607101]

Leising KJ, Garlick D, Blaisdell AP. Overshadowing by proximity with pigeons in an automated openfield and touchscreen. Journal of Experimental Psychology: Animal Behavior Processes. 2011; 37(4):488-494. [PubMed: 21604908]

Leising KJ, Garlick D, Parenteau M, Blaisdell AP. Behavioral research in pigeons with ARENA: An automated remote environmental navigation apparatus. Behavioural Processes. 2009; 81:105-113. [PubMed: 19429204]

Mackintosh NJ. Do not ask whether they have a cognitive map, but how they find their way about. Psicologica. 2002; 23:165-185.

Miller NY, Shettleworth SJ. Learning about environmental geometry: An associative model. Journal of Experimental Psychology: Animal Behavior Processes. 2007; 33:191-212. [PubMed: 17620021]

Moore JW, Schmajuk NA. Kamin blocking. Scholarpedia. 2008; 3(5):3542.

O'Keefe, J.; Nadel, L. The hippocampus as a cognitive map. Oxford: Oxford University Press; 1978.

Rodrigo T, Chamizo VD, McLaren IPL, Mackintosh NJ. Blocking in the spatial domain. Journal of Experimental Psychology: Animal Behavior Processes. 1997; 23:110-118. [PubMed: 9008865]

Rodriguez CA, Chamizo VD, Mackintosh NJ. Overshadowing and blocking between landmark learning and shape learning: The importance of sex differences. Learning and Behavior. 2011; 39(4):122-135.

Rodriguez CA, Chamizo VD, Mackintosh NJ. Do hormonal changes appear at the onset of puberty determine the strategies used by female rats solving a navigation task? Hormones and Behavior. 2013; 64(1):122-35. [PubMed: 23732821]

Sawa K, Leising KJ, Blaisdell AP. Sensory preconditioning in spatial learning using a touch screen task in pigeons. Journal of Experimental Psychology: Animal Behavior Processes. 2005; 31:368375. [PubMed: 16045391]

Spetch ML, Mondloch MV. Control of pigeons' spatial search by graphic landmarks in a touch-screen task. Journal of Experimental Psychology: Animal Behavior Processes. 1993; 19:353-372.

Spetch ML, Cheng K, Mondloch M. Landmark use by pigeons in a touch-screen spatial search task. Animal Learning \& Behavior. 1992; 20:281-292.

Spetch ML. Overshadowing in landmark learning: Touch-screen studies with pigeons and humans. Journal of Experimental Psychology: Animal Behavior Processes. 1995; 21:166-181. [PubMed: 7738499]

Spetch ML, Cheng K, MacDonald SE. Learning the configuration of a landmark array: I. Touch-screen studies with pigeons and humans. Journal of Comparative Psychology. 1996; 110:55-68. [PubMed: 8851553]

Spetch ML, Cheng K, Mondloch MV. Landmark use by pigeons in a touch-screen spatial search task. Animal Learning \& Behavior. 1992; 20:281-292.

Spetch ML, Cheng K, MacDonald SE, Linkenhoker BA, Kelly DM, Doerkson SR. Use of landmark configuration in pigeons and humans: II. Generality across search tasks. Journal of Comparative Psychology. 1997; 111:14-24.

Stahlman WD, Blaisdell AP. Blocking of spatial control by landmarks in rats. Behavioural Processes. 2009; 81:114-118. [PubMed: 19429205] 
a.

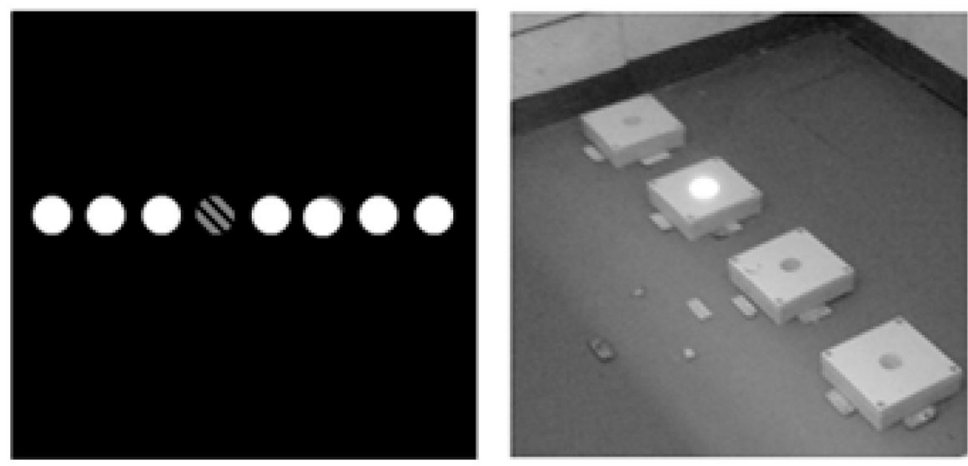

b.

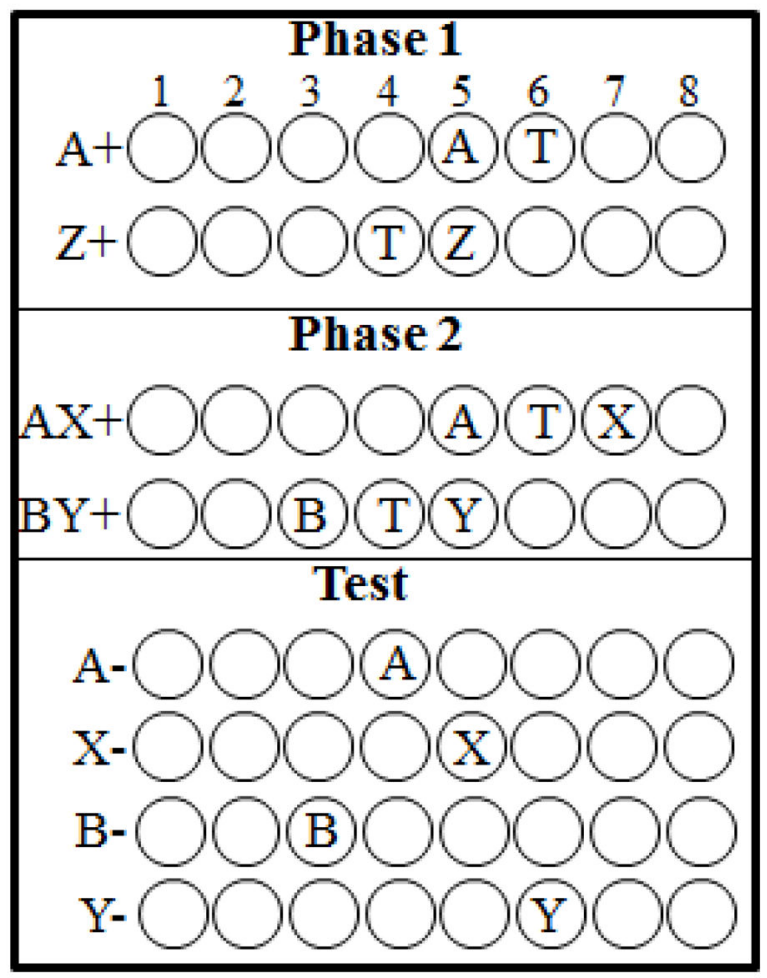

Figure 1.

a) Left-hand panel is an example of the display used for the touchscreen spatial task in Experiment 1a. The eight response units are shown, including one landmark at position four. The right-hand panel is a photo of four (of six) ARENA modules in the test area (reprinted with permission from Leising et al., 2011) in Experiment 1b. b) A schematic of the experimental design. The letters "A", "X", "Y", and "B" denote different colored landmarks and " $\mathrm{T}$ " indicates the location of the target. The use of "+" indicates the opportunity for reinforcement whereas the use of "-" indicates no opportunity for reinforcement. Numbers along the top denote unit positions along the linear array of eight units (Experiment 1a) or six (Experiment 1b) modules. Figures are not drawn to scale. 


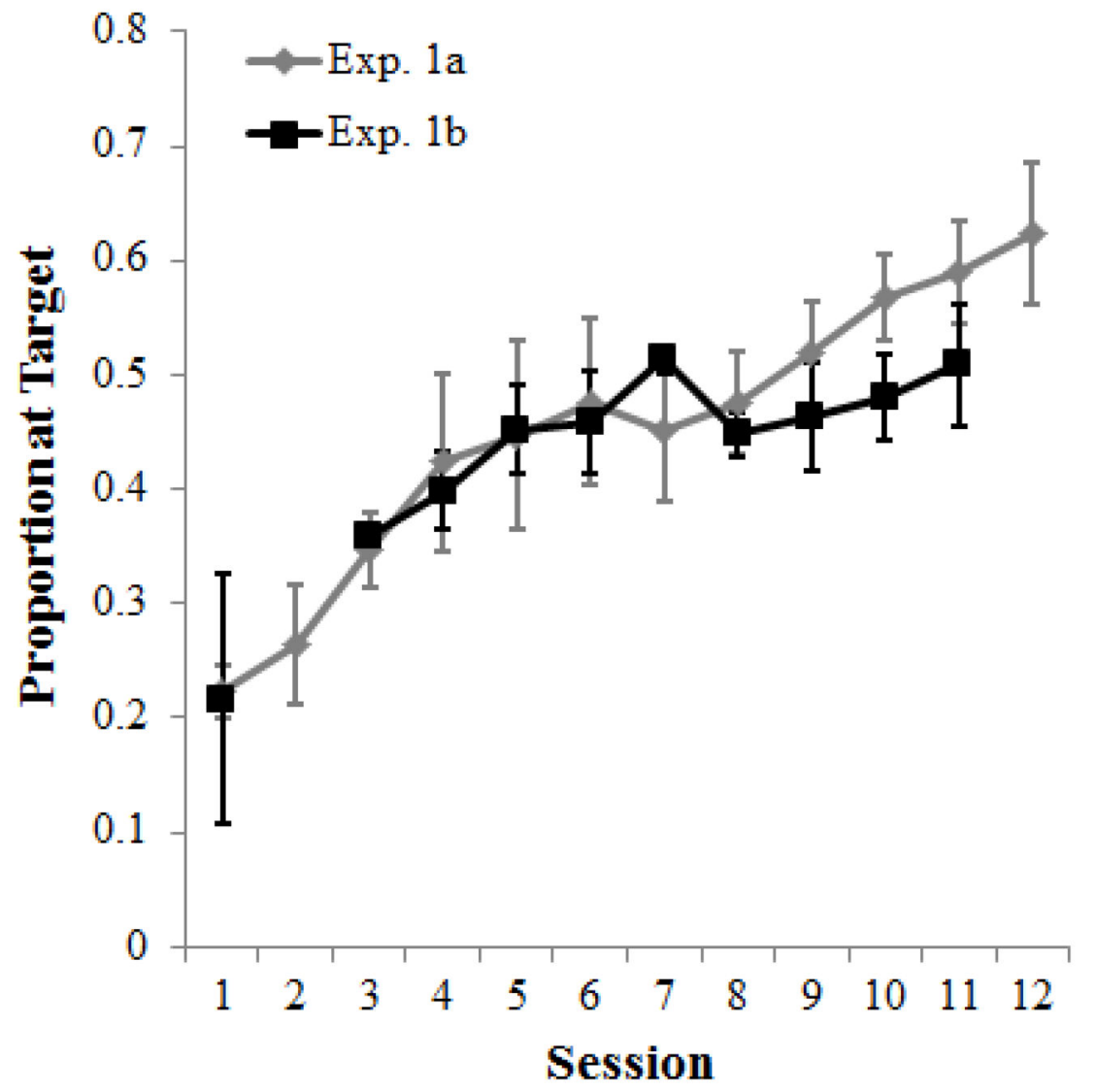

Figure 2.

Mean proportion of responding to the predicted target on trials of LM A and Z across sessions of Phase 1 for subjects in the touchscreen (Experiment 1a) and ARENA (Experiment 1b). Only sessions in which all subjects contributed data are displayed for each experiment. Error bars represent the standard error of the mean. 

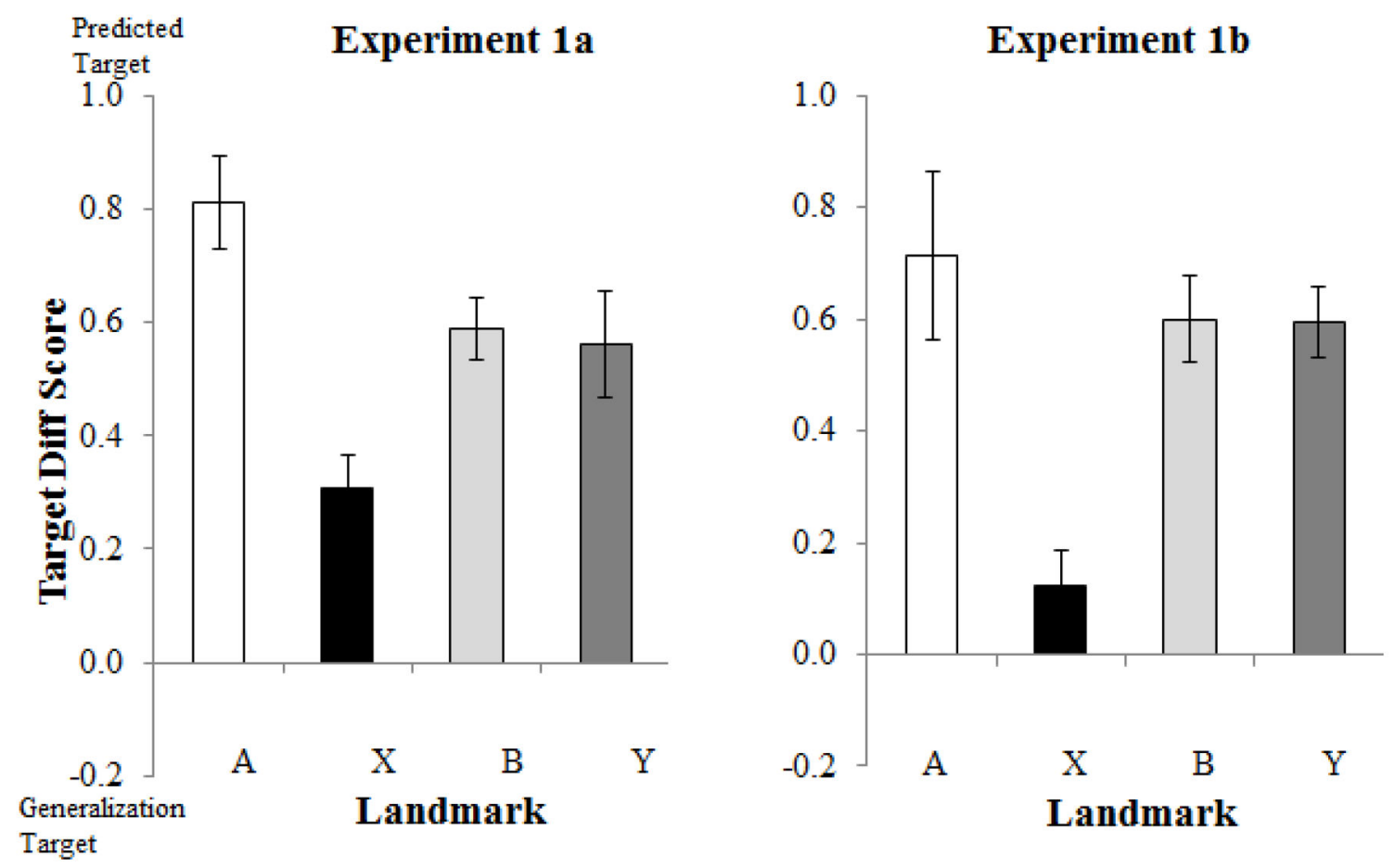

Figure 3.

Mean difference in the proportion of responding to the predicted target and generalization target during testing for each landmark in Experiment 1a (left panel) and experiment $1 \mathrm{~b}$ (right panel). Error bars represent the standard error of the mean. The absolute value of the difference indicates the strength of spatial control, whereas, the sign indicates the preference to respond at the predicted or generalization target. 
a.

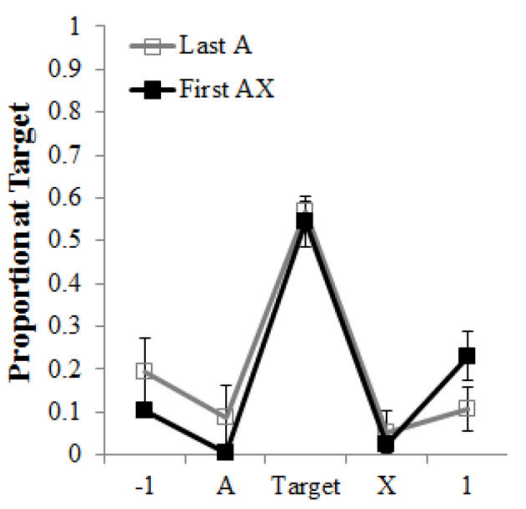

b.

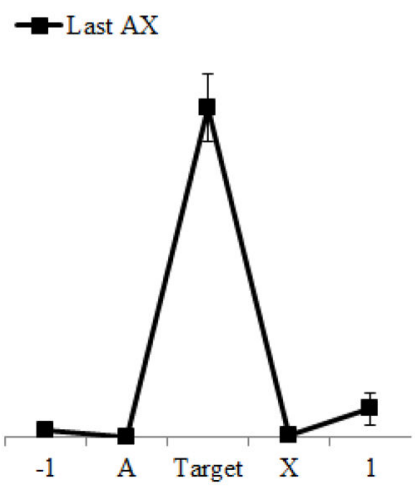

Figure 4.

a) Mean distribution of searches across the target location and the two response units to the left and right of the target on each trial. The number of responses at each unit was standardized across trials, such that responses were recorded relative to the expected target on each trial. The response units occupied by landmarks during each trial are indicated in the $\mathrm{X}$-axis; the units to the left and right of the landmarks on each trial are labeled as -1 (to left of the target and landmark) and 1 (to the right of the target and landmark), respectively. Data are from the last A+ trial of Phase 1 and the first AX+ trial of Phase 2 in ARENA during Experiment 1b. b) Mean distribution of searches across the target locations and the two response units to the left and right of the target during the last $\mathrm{AX}+$ trial of Phase 2 in ARENA during Experiment $1 b$. 